\title{
Review-Artikel: Zum aktuellen Stand des Plattformkapitalismus
}

\section{Zusammenfassung}

Die Plattform ist in den letzten Jahren sowohl eine zentrale Institution als auch wesentliche Metapher der gegenwärtigen Verfasstheit westlicher Gesellschaften geworden, so dass von einem „Plattformkapitalismus“ die Rede ist. Nachhaltige Auswirkungen zeigen sich in verschiedenen Sphären - darunter besonders zentral in der Organisation von Arbeit. Nach einer breiten medialen Thematisierung spiegelt sich das Phänomen auch in einer zunehmenden wissenschaftlichen Auseinandersetzung wider. Im folgenden Beitrag werden vier aktuelle Werke vorgestellt und diskutiert, die den englischsprachigen Diskurs wiedergeben.

Schlagwörter: Plattformkapitalismus, Digitalisierung, Zukunft der Arbeit, Organisierung von Arbeit, Crowdwork

\section{Review Article: The Current Situation of Platform Capitalism}

\section{Abstract}

In recent years, the platform has become a pivotal institution as well as an essential metaphor of western societies' constitutions. Subsequently, the term "platform capitalism" has been created. The enduring consequences appear in different spheres - especially in the organisation of work. Having firstly become a key theme in the media, the phenomenon is now the subject of a growing scientific debate. In the following article, four current books are presented and discussed, each reflecting the discourse on the subject platforms in the English-speaking world.

key words: platform capitalism, digitalisation, future of work, organisation of work, crowdwork (JEL: J40, O33, P12)

* Heiner Heiland, Technische Universität Darmstadt, Institut für Soziologie, Dolivostraße 15, D-64293 Darmstadt. E-Mail: heiland@ifs.tu-darmstadt.de

** Artikel eingegangen: 1.11.2017; revidierte Fassung akzeptiert: 13.3.2018. 
Dyer-Whiteford (2015). Cyber-Proletariat. Global Labour in the Digital Vortex. London: Pluto Press.

Scholz, Trebor (2017). Uberworked and Underpaid. How Workers are Disrupting the Digital Economy. New York: Polity Press.

Srnicek, Nick (2017). Platform Capitalism. Cambridge, UK: Polity Press.

Sundararajan, Arun (2016). The Sharing Economy. The End of Employment and the Rise of Crowd-Based Capitalism. Cambridge, MA: MIT Press.

\section{Differente Perspektiven}

Die Geschichte ist bekannt: Der Welt größtes Taxiunternehmen, Uber, besitzt keine Fahrzeuge; der Welt größter Anbieter von Übernachtungen, Airbnb, verfügt über keine eigenen Zimmer; und der Welt populärster Medienanbieter, Facebook, schafft keine eigenen Inhalte (Goodwin, 2015). ${ }^{1}$

Das Phänomen wurde bereits als der nächste „tech gold rush“ (Wohlsen, 2013) gehandelt und als „Plattformkapitalismus“ (Lobo, 2014) gekennzeichnet. Denn es sind oft noch junge, aber hoch bewertete Online-Plattformen, die innerhalb weniger Jahre lang etablierte ökonomische Strukturen und komplexe Wertschöpfungsketten transformiert haben. Und mit der üblichen Verspätung reagieren auch die Sozialwissenschaften auf diese Entwicklungen, so dass zunehmend mehr Veröffentlichungen zu dieser breiten Thematik erscheinen, von denen im Folgenden vier zentrale Werke aus dem englischsprachigen Diskurs besprochen werden.

Die Bücher unterscheiden sich markant durch die theoretischen Hintergründe ihrer Verfasser, ihre Foki sowie die erwarteten Entwicklungen und empfohlenen Eingriffe. Gemein ist ihnen die Auseinandersetzung mit den Folgen digital unterstützten Wirtschaftens für ökonomische und soziale Strukturen. Insbesondere das Konzept der Plattform steht dabei im Fokus.

Versteht man wissenschaftliche Werke im Anschluss an Pierre Bourdieu (1999) als Positionierungen innerhalb eines wissenschaftlichen Feldes, in dem um Kapitalien, Macht und Deutungshoheit gerungen wird, so befindet sich Nick Dyer-Whiteford mit seiner dezidiert marxistischen Perspektive auf den (weltumspannenden) digtalen Vortex des Kapitals auf der weniger autonomen Seite des Kontinuums. Auch Nick Srnicek ist stärker an diesem heterodoxen Pol des Feldes zu verorten, setzt er sich doch mit anderer Anlehnung an Marx kritisch mit der Ressource Daten als dem „,neuen Öl“ auseinander und analysiert Grundlage, Geschäftsmodell und Zukunft sehr diverser Arten von Plattformen, die für ihn den neuartigen Plattformkapitalismus ausmachen. Trebor Scholz Perspektive ist enger auf die arbeitsvermittelnden Plattformen gerichtet. Obwohl er sich der kritischen Analyse dieser Phänomene anschließt, hofft er, deren Techniken emanzipatorisch anwendbar machen zu können

1 Mittlerweile hat Uber über mehrere Tausend Autos gekauft mit dem Ziel, autonomes Fahren voranzutreiben. Airbnb plant den Bau von Wohnungen und Facebook investiert in die Produktion eigener TV-Sendungen. Trotz allem ist mit diesem Zitat weiterhin die Logik des Phänomens prägnant identifiziert. 
und liefert hierzu auch die notwendige Blaupause. Das autonome Ende des Spektrums belegt Arun Sundararajan mit seinem affirmativen Buch zur „Sharing Economy“. In diesem sieht er auf Basis der bereits von Scholz thematisierten Plattformen eine viel versprechende neue Form des ,crowd-based capitalism“ entstehen.

Allen Büchern gemein ist demnach eine Auseinandersetzung mit neuen, digitalen Formen der Arbeit und des Wirtschaftens. Dabei sind der Zugang und auch die theoretische Perspektive eine jeweils sehr unterschiedliche, die von marxistischen bis hin zu eher neoklassischen ökonomischen Ansätzen reichen. Fernab dieser Unterschiede teilen die Texte aber auch einen empirischen Mangel. So verweisen alle Autoren - wenn überhaupt - nur auf begrenztes eigenes empirisches Material, das die umfassenden vertretenen Thesen unmittelbar belegt. Doch trotz allem bietet die Lektüre der Bücher wichtige Anregungen für ein Verständnis und eine Diskussion der ,Zukunft der Arbeit“.

\section{Die Sharing Economy als „crowd-based capitalism”}

Sundararajan ist einstiger Unternehmer der New Economy, seine Forschung wird von Google, IBM und Microsoft unterstützt. Er gilt als besonders sichtbarer öffentlicher Intellektueller der Sharing Economy, der sowohl den US-amerikanischen Kongress als auch das Europäische Parlament berät. Sein hier besprochenes Buch, The Sharing Economy, ist mehrfach preisgekrönt, als Hörbuch erschienen und wird als ,,path-breaking“ gepriesen. Doch aus (insbesondere arbeits-) soziologischer Perspektive ist Kritik an seinen Thesen angebracht.

$\mathrm{Zu}$ Beginn konstatiert Sundararajan einen radikalen gesellschaftlichen Wandel, der zwar eine tiefgreifende Disruption bewirke, doch als Vorbote neuer Wohlstandsgenerierung zu verstehen sei (2016, S. 2). Kern dieser Transformation ist dem Autor zufolge die durch Plattformen verursachte Ablösung des managerialen Kapitalismus durch eine neue „crowdbasierte“ Form (S. 69). Ihren Ursprung hätten die Plattformen in den jüngsten digitalen technischen Innovationen und deren umfassender Verbreitung - namentlich Smartphones sowie mobiler Internetanbindung (S. 47). Mittels der damit einhergehenden Möglichkeiten können nun bisher marginalisierte ökonomische Modelle mit klassischen kapitalistischen verbunden werden, so Sundararajan. Demnach sei es mit Hilfe der neuen vermittelnden Plattformen möglich, bisher nur gering genutzte physische Ressourcen zu teilen und damit ihre Auslastung zu vergrößern. In der Folge entstehe - so der Autor im Anschluss an bspw. Yochai Benkler (2004) - ein idealer Markt mit sozialen Signalen und Motivationen anstatt Preisen zur Generierung von Informationen und Steuerung von Handlungen (Sundararajan, 2016, S. 32).

Sundararajan stellt fest, dass der Kapitalismus des 20. Jahrhunderts ineffizient, unpersönlich und kommerziell geworden sei, doch diese Entwicklung könne durch Plattformen und die damit einhergehenden auf Reziprozität basierenden Kulturen des Schenkens (,gift economies") überwunden werden (S. 35). Was man mit Polanyi als eine Wiedereinbettung des Kapitalismus in das Soziale verstehen könnte, geht Sundararajan zufolge insbesondere auf die „Digitalisierung des Vertrauens“ zurück (S. 60). Damit ist erstens das Aufkommen einer Form digitalen sozialen Kapitals gemeint, das bspw. durch Facebook sichtbar und 
damit auch zur Verifikation der Identität der NutzerInnen auf anderen Plattformen verfügbar wird. ${ }^{2}$ Zweitens ist darüber hinaus mit der Option, sich gegenseitig zu bewerten, eine retrospektive Sanktionsmöglichkeit geschaffen, die erlaubt, sich eine Reputation aufzubauen. Diese Entwicklung erlaubt es laut Sundararajan, dass ursprünglich lokal und sozial eng begrenzte „close-knit communities“ expandieren (S. 38) und dass einander gänzlich Fremde miteinander interagieren. Dabei sieht er die Möglichkeit, mit einer dadurch steigenden „,connectedness“ zentrale Probleme von Gewalt bis hin zu Bildungsfragen zu lösen oder zumindest zu mildern (S. 45).

Sundararajans Ausführungen laufen der soziologischen Erkenntnis zuwider, der zufolge selbst symbolische Kulturen des Schenkens nicht einem do ut des entsprechen, sondern ihnen strukturelle und vermachtete Aspekte inhärent sind (Bourdieu, 1998, S. 139). Dagegen spricht außerdem, dass tatsächliches Teilen als explizit nichtkapitalistisches Handeln und mittels Geld vermittelter Warenaustausch einander widersprechen (Belk, 2010; 2014). Darüber hinaus kann die Betonung der zunehmenden Relevanz von ,non-price-based social relations“ (Lessig, 2009, S. 146) anstatt als Wiedereinbettung vielmehr als Landnahme und fortschreitende Kommodifizierung verstanden werden. Die Sharing Economy transzendiert demnach nicht die gesellschaftliche Form, sondern weitet diese auf zuvor nicht direkt kapitalistisch organisierte Felder aus. Eine Taxifahrt ist - auch und insbesondere wenn sie per Plattform vermittelt und von Solo-Selbstständigen ausgeführt wird -kein Austausch zwischen Freien und Gleichen. Und ist der zukünftige Erfolg der Fahrenden direkt von der Leistungsbewertung abhängig, so gewinnen Aspekte emotionaler Arbeit an Relevanz und erhöhen die Anforderungen an die Arbeitenden (Hochschild, 1983; Penz \& Sauer, 2016).

Auch Sundararajans Hoffnungen auf die Dezentralität des Internets und ein damit verbundenes Ende eines Informationspanoptismus, in dem Wissen lediglich im Zentrum einer Person oder Gruppe zur Verfügung steht, dürfte allenfalls begrenzt realistisch sein. So zeigt sich nicht nur beim Zugang zum Internet je nach Nationalität und Region ein „digital gap“, sondern besonders auch in der Art der Nutzung, die deutliche Klassendifferenzen aufweist (Dudenhöffer \& Meyen, 2012). Und eine solche zeigt sich auch betreffend die Sharing Economy (Schor et al., 2016). Darüber hinaus weisen plattformkapitalistische Unternehmen bis dato in erster Linie eine panoptische Struktur auf, die es ihnen erlaubt, trotz der Auslagerung der Arbeitskräfte umfassende Kontrolle über die Arbeitenden und deren Tätigkeiten ausüben zu können (Heiland, 2018).

Sundararajan hält die Effekte der Sharing Economy bisher für nur schwer messbar. Er geht allerdings davon aus, dass man erst am Anfang stehe und Plattformen das Potenzial mit sich brächten, zentrale ökonomische Ungleichheitsprobleme zu reduzieren (Sundararajan, 2016, S. 129). So stimmt er Thomas Pikettys These des strukturellen Wachstums ökonomischer Ungleichheit zu, doch sieht er im „,crowd-based capitalism“ die Möglichkeit für alle gegeben, mittels Plattformen auch noch so geringes Kapital zu investieren und von den Rückflüssen zu profitieren (S. 123). Dies geht allerdings am Argument Pikettys vorbei, der

2 Was zuvor nur als Kredit-Scores oder der Schufa zur Verfügung stand, nimmt auf diesem Weg neue und umfassendere Formen an. Neben einem Facebook-Account erstellt die ,general-purpose portable reputation“ Plattform Traity bspw. ein ,holistischeres“ Profil der Nutzenden, basierend auf Bewertungen auf anderen Plattformen, diversen Accounts sozialer Online-Netzwerke und auch offiziellen Verifikationen wie dem Personalausweis (S. 64). 
auf die strukturelle Diskrepanz zwischen den verschiedenen Einkommen abhebt, die einen marktbasierten Ausgleich unmöglich macht. Darüber hinaus zeigt sich in der Empirie, dass Arbeit vermittelnde Plattformen bestehende Ungleichheiten nicht verringern, sondern verstärken (Schor, 2017).

In der kontroversen Frage der Regulation des Plattformkapitalismus vertritt der Autor eine Position, die die Vorteile einer weit gehenden Liberalisierung betont. So böten die Plattformen mit ihrer Vermittlungstätigkeit zwischen verschiedenen Gruppen von NutzerInnen neue Lösungsmöglichkeiten für das Problem des Marktversagens (S. 140). Dabei spricht sich Sundararajan für eine - wenn überhaupt - reaktive Regulation von Plattformökonomien aus und fordert darüber hinaus gehend „safe harbors“, die es den Plattformen erlauben, sich zu entwickeln und nicht von vorauseilenden und womöglich unpassenden Regularien erdrückt zu werden. In den allgemein üblichen Bewertungssystemen der Plattformen, die meist sowohl eine Evaluation der Arbeitenden als auch der KundInnen ermöglichen, sieht er einen neuartigen und marktgesteuerten Kontrollmechanismus, der Regulationen weitgehend überflüssig macht (S. 148; ders., 2014).

Es bleibt, dass Sundararajan einen kenntnisreichen Überblick über die jüngeren Entwicklungen im Bereich der Sharing Economy gibt. Dabei macht er aus einem etwaigen „,capital-leaning bias“ keinen Hehl (S. 27) und kommt daher zu einer idealistischen und in der Folge auch einseitigen Perspektive auf das Phänomen. Bestehende ökonomische und soziale Strukturen und Beziehungen bleiben in der Analyse willfährig vernachlässigt. Infolgedessen ist seine Hoffnung trügerisch, dass gegenwärtig schwächelnden Ökonomien vermögen von einer in erster Linie durch Plattformen initiierte Stimulation zu prosperieren. Denn gegenwärtige gesamtökonomische Mängel sind eher auf eine begrenzte Nachfrage zurückzuführen, deren Elastizität sich auch durch Digitalisierung nicht zu ändern vermag (Staab, 2017). Dass Sundararajan dergleichen nicht in Betracht zieht, ist auf seine markante Ignorierung der Arbeitsseite des ,crowd-based capitalism“ zurückzuführen. Denn Arbeit im Plattformkapitalismus stellt sich für ihn nur als Chance für die „Beschäftigten“ dar und somit auch als „Mittelweg zwischen Kapitalismus und Sozialismus“ (2016, S. 44).

\section{Kritik am und Alternativen zum Plattformkapitalismus}

Dass es sich bei Plattformkapitalismus weniger um eine humanere Gestaltung gesellschaftlicher Verhältnisse handelt, sondern stattdessen um eine radikalisierte Ökonomisierung, die mit prekärer Arbeit einher geht, wird von Trebor Scholz vertreten. Dessen Buch Uberworked and Underpaid ist zweigeteilt und stellt nach einer Analyse digitaler Arbeit die Konzeptionalisierung einer Alternative in Form kooperativ organisierter Plattformen vor. Anders als Sundararajan plädiert Scholz, der sich selbst als Wissenschaftler und auch als Aktivist versteht, damit sowohl für eine weitgehende Regulierung der neuen Arbeit vermittelnden Plattformen als auch für eine Marktsteuerung, die auf von Arbeitern und Arbeiterinnen geführten Plattformen fußt.

Ausgangspunkt ist für Scholz eine Analyse, wie neue Formen der Wertextraktion mittels des Digitalen vonstattengehen. Damit nimmt er ein weites Feld - von freiberuflichen Web Designern bis zu programmierenden Computerspielerinnen, von Taxifahrern bis hin 
zu Facebooknutzerinnen - in den Fokus und versucht sich an einer Typologisierung solch diverser Formen digitaler Arbeit. Im Anschluss schlussfolgert er, dass traditionelle Formen der Ausbeutung mittels des Digitalen weit weniger zum Tragen kommen. Als Konsequenz prägt er den Begriff des „Crowd Fleecing“, mit dem er die neuen und weit weniger sichtbaren Arten der Ausbeutung bezeichnet (2017, S. 113).

Scholz widmet sich dem Phänomen digitale Arbeit kenntnisreich und umfassend, doch es zeigt sich die Herausforderung, die die Analyse digitaler Plattformen mit sich bringt. Denn wie bereits bei Sundararajan werden sehr diverse Arten von Arbeit und deren Organisation unter den gemeinsamen Nenner der Plattform subsumiert. Seine panoramische Typologisierung führt dazu, dass das Besondere unter ein sehr weit gefasstes Allgemeines geordnet wird und damit die Spezifika der vielen verschiedenen Plattformen aus dem Blick geraten bzw. ihre vermeintlichen Gemeinsamkeiten nicht klar erkennbar, doch mit dem recht unpräzisen Begriff des „Crowd Fleecing“ zusammengefasst werden. Dabei liegt der eigentliche Fokus und auch die Stärke von Scholz Analyse auf den Arbeit vermittelnden Plattformen, deren Spektrum durch die Unternehmen Amazon Mechanical Turk und Uber, sprich die lokal ungebundene bzw. gebundene Plattformarbeit, abgesteckt ist. Und dies ist auch der primäre Fokus der von Scholz im Anschluss an seine Analyse aufgezeigten Alternativen.

Denn keineswegs weist er Plattform-Konzepte als solche in ihrer Gänze zurück. Stattdessen strebt er eine alternative Nutzung dieses Organisationskonzepts und seiner Techniken an, was mittlerweile unter dem Namen Plattform-Kooperativismus über einige Anhängerinnen und Anhänger verfügt. Und zahlreiche vorgestellte internationale Beispiele zeigen, dass es sich beim gemeinschaftlichen Eigentum an Plattformen sowie deren Ausgestaltung um mehr als eine fixe Idee handelt. Die Umsetzung weiterer kooperativer Plattformen wird von Scholz und anderen voran getrieben und durch die Bereitstellung eines digitalen Ökosystems für solche Initiativen aktiv unterstützt. Doch einige Herausforderungen bleiben bestehen; auch gegenüber der Vorstellung einer umfassenden Verwirklichung ist Skepsis angebracht. Das Wagnis nichtkapitalistischer Kooperativen in einer kapitalistischen Umwelt wurde bereits von Rosa Luxemburg thematisiert (1899 [1982]), was auch Scholz anerkennt und diskutiert. Darüber hinaus zeigt sich, dass im Bereich der tatsächlich teilenden Sharing Economy zahlreiche genderbasierte, ethnische und Klassenungleichheiten reproduziert werden (Schor et al., 2016), und somit die Gefahr besteht, einen „luxury cooperativism“ zu kreieren (Duda, 2016; Sassen, 2016).

Scholz bietet eine kritische und fundierte Analyse digitaler Arbeit. Dabei bettet er die Analyse der Entstehung des Plattformkapitalismus in eine Makro-Perspektive ein, so dass Plattformen nicht allein als Ergebnis technischer Innovationen erscheinen. Digitalisierung stellt damit eine notwendige, aber nicht eine hinreichende Bedingung für eine Kommodifizierung der Arbeit dar. Die neuartige Regulation der Arbeitsbeziehungen erweist sich demzufolge als zentraler Aspekt.

Das Buch tangiert zahlreiche digitale Arbeitsphänomene und bietet einen breiten und gewinnbringenden Überblick. Doch die Breite der Perspektive schränkt mitunter die Tiefe der Analyse ein, so dass die Gemeinsamkeit von online vermittelter Arbeit und der Inwertsetzung von Aufmerksamkeit und Aktivität von Plattformnutzerinnen und -nutzern nicht immer auf der Hand liegt. So stellt sich die Frage, ob bei allen Tätigkeiten, die Scholz als 
digitale Arbeit identifiziert, tatsächlich die gleichen Kämpfe ausgefochten werden oder ob diese nicht doch disparat sind. Der zweite Teil des Buches beinhaltet sowohl einen begründeten Diskursanstoss als auch den Entwurf eines politischen Projekts. Dieses ist weniger als umfassende Transformationsperspektive aufzufassen als vielmehr als eine ambitionierte Intervention, die hofft, realistische Alternativen zu verwirklichen, die eine faire und demokratische Organisation von Arbeit ermöglichen.

\section{Daten als das „neue Öl“ und ihre Ausbeutung durch Plattformen}

Plattformen in einem weiteren Sinn stehen auch im Fokus von Nick Srniceks Buch Platform Capitalism (2017), das im März auch auf Deutsch erschienen ist. Srnicek, zuletzt als Vertreter des Akzelerationismus (Srnicek \& Williams, 2013) sowie Theoretiker des Postkapitalismus (dies., 2015) hervorgetreten, bietet auf Basis einer weit reichenden Typologisierung einen Überblick über die unterschiedlichen Anwendungen einer Plattformlogik und legt darüber hinaus die makroökonomischen Hintergründe in Form der Finanz- und Wirtschaftskrise 2008ff. dar.

Gegenstand der Betrachtung sind sehr diverse Plattformen, deren Gemeinsamkeit die Bereitstellung eines Interaktionsraums für verschiedene Gruppen darstellt (Srnicek, 2017, S. 43). Das demzufolge sehr breit gefasste Spektrum an Plattformen differenziert Srnicek in fünf Typen mit spezifischen Foki: Werbung (exemplarisch Google, Facebook), Cloud (Amazon Web Services), Industrie (GE, Siemens), Produkte (Rolls Royce, Spotify) und ,lean platforms“"(Uber, Airbnb) (S. 49).

Alle diese diversen Unternehmen beruhen laut Srnicek auf Plattformen und damit auf einem dem digitalen Zeitalter adäquaten Geschäftsmodell. Demnach stellen diese Plattformen nicht nur Informationen, Produkte o.ä. zur Verfügung, sondern stattdessen in erster Linie zunehmend basale Infrastrukturen (Gillespie, 2010). Gemeinsam ist ihnen darüber hinaus die zentrale Absicht, große Mengen an Daten von den Nutzenden zu sammeln, die als „neues Öl“ das Rohmaterial ihres Geschäftsmodells darstellen (Srnicek, 2017, S. 39). Srnicek betont dabei den trivialen Aspekt, dass auch hierbei notwendigerweise profitorientiertes Handeln im Fokus steht. Aufgrund dessen sowie infolge ihrer organisationalen Spezifik neigen Plattformen dazu, die Gewinnung von Daten auszuweiten, ihre Ökosysteme zu schließen und dabei als Gatekeeper zu fungieren sowie Märkte zu monopolisieren (S. 98).

Auf dieser Basis nimmt der Autor eine detailliertere Betrachtung der zuvor dargelegten fünf Plattformtypen und den unter diesen stattfindenden und ausstehenden ,great platform wars" vor. Gegen den sonst üblichen Tenor geht er dabei von engen Grenzen des Modells der „lean platform“ aus, das bei Sundararajan und Scholz noch als Zukunft der Arbeit im Fokus steht. Diese Geschäftsmodelle stellen Srnicek zufolge einen Rückschritt in frühere Stadien der Internetökonomie dar, in denen ,Wachstum vor Profit ${ }^{`}$ zur Dotcom-Blase der 1990er führte (S. 75). Denn im Kern weiten sie einzig bereits bestehende OutsourcingTrends aus und bleiben bis heute den Nachweis ihrer Profitabilität schuldig (S. 87). Dabei ist allerdings fraglich, inwieweit Srnicek mit Uber seine Schlüsse nicht zuallererst aus einer zwar radikalen, aber weniger repräsentativen Form dieses Modells zieht. Auf der anderen Seite stehe als aussichtsreichste Plattform Amazon, die mit ihrem breiten und basalen An- 
gebot Chance habe, sowohl Google als auch Facebook hinter sich zu lassen, da letztere bereits an die Grenzen ihres werbebasierten Modells gerieten (S. 126). Abschließend äußert sich Srnicek verhalten gegenüber kooperativen Ansätzen, wie Scholz sie propagiert. Er votiert stärker für ein staatliches Eingreifen im Rahmen aktualisierter Kartellrechte oder gar für Vergesellschaftungen zentraler Plattformen (S. 127).

Srnicek legt einen profunden Überblick betreffend des Aufkommens sowie der aktuellen Entwicklungen der Plattformlogik vor. Gewinnbringend ist dabei die makroökonomische Perspektive. Denn anhand dieser zeigt sich, dass Formen plattformvermittelter Arbeit weder grundsätzlich neu sind, noch lassen sie sich allein durch das Aufkommen technischer Innovationen erklären (Stanford, 2017). Wo Sundararajan noch mit einer technizistischen Perspektive auf die Ursache der Plattformen aufwartet, erscheinen diese bei Srnicek als Teil einer kontinuierlichen Entwicklung, die auf einer losen Geldpolitik sowie der Suche des Finanzkapitals nach ertragreichen Investitionsmöglichkeiten in Zeiten niedriger Zinsen beruht (Kapitel 1). Doch die unweigerlich gröbere Analyse ist mitunter holzschnittartig und lässt es an einer tiefer gehenden Betrachtung fehlen, weswegen das Buch eher als Überblick und Diskussionsanstoß zu verstehen ist. So wäre neben den Logiken der spezifischen Plattformen und Sektoren zu diskutieren, inwieweit primär Daten im Fokus plattformbasierter Geschäftsmodelle stehen. Und ebenso müsste weiterhin Gegenstand der Diskussion sein, inwieweit die Tätigkeiten der besagten Plattformen tatsächlich als Ausbeutung zu verstehen sind (Fuchs, 2014; Sevignani, 2017) und was dies für die Betroffenen bedeutet.

\section{Globale Perspektiven auf ein entstehendes Cyber-Proletariat}

Die Folgen für die Betroffenen in einer sehr weit gefassten Form stehen im Fokus Nick Dyer-Whitefords Buch zum Cyber-Proletariat (2015). Jenes schließt an seine Studie zur Aktualität des Marx'schen Werks in Zeiten des Digitalen an, Cyber-Marx (1999). Doch während der Autor zuvor im Zuge seines Anschlusses an post-operaistische Theorien, die die Gestaltungskraft der Arbeitenden betonen, optimistischer gestimmt war, ergänzt er diese Sicht nun durch Ansätze der Kommunisierung, für die die Arbeitenden originärer Teil der Kapitalseite sind. Wie der Autor selbst darlegt, hat er in der Folge eine skeptischere Sichtweise eingenommen. Diese begründet er mit der ihm zufolge zunehmend globalen und kybernetischen Struktur des gegenwärtigen Kapitalismus. Angesichts dieser Diagnose strebt der Autor an, die Klassenzusammensetzung der Gegenwart zu betrachten.

Das globale kapitalistische System wird dabei als ein Sturm versinnbildlicht, der , alles Stehende und Ständische " um ihn herum vereinnahmt und beeinflusst und damit auch vermeintlich unabhängige Phänomene verbindet. So deckt Dyer-Whiteford zahlreiche Verbindungen zwischen einander fernen Ereignissen auf. Einleitend konstatiert er bspw. eine indirekte Beziehung zwischen der Ersetzung von Menschen durch einen Investitionsalgorithmus im Vorstand eines Unternehmens für Risikokapital mit einer am gleichen Tag stattfindenden Explosion in einer bestreikten türkischen Kohlemine (S. 1-3). Die durch den Algorithmus beeinflussten Entscheidungen bewirkten dabei keineswegs direkt das Unglück, doch besteht dem Autor zufolge ein Zusammenhang zwischen der digitalen Ökonomie und deren meist ignorierten, aber grundlegenden materiellen Schattenseite. 
Das Vorhaben des Buches gleicht David Harveys Analyse. Letzterer beschreibt seine eigene Arbeit als eine Kartierung des globalen Kapitals, die einer Wetterkarte ähnelt, auf der sich einander beeinflussende Hoch- und Tiefdruckgebiete mit vereinzelten Stürmen sichtbar werden. Dabei wird eine Struktur erkennbar, die trotz aller Bewegung persistente Ungleichheiten aufweist (Harvey, 2010, S. 153).

Dyer-Whiteford betont die zentrale Relevanz der Klasse. Das Cyber-Proletariat bezeichnet für den Autor nicht nur die direkt mit dem Digitalen verbundenen Arbeitenden, sondern alle Personen, die von dessen Wertschöpfungsprozessen, der zugrunde liegenden Ressourcengewinnung sowie der verborgenen Reproduktionsarbeiten in irgendeiner Art beeinflusst sind. Diese Personen werden infolge einer ,globalen Proletarisierung“ (Karl Heinz Roth) sowie einer „Entagrarisierung der Welt“ (Immanuel Wallerstein) zahlreicher und infolge der Kybernetisierung wird ihre Ausbeutung intensiviert (Dyer-Whiteford, 2015, S. 132). Doch zugleich, so Dyer-Whiteford, entsteht damit ein verknüpftes Surplus an Menschen, die ihren Unmut in neuen Klassenkämpfen ausdrücken, wie sich besonders 2011 während des arabischen Frühlings sowie verschiedener Aufstände in New, York, London, Athen und anderen Orten zeigte (S. 147). Dies dient dem Autor als Grundlage für die Analyse eines weiteren taktischen Vorgehens (S. 168).

Dyer-Whiteford füllt mit seinem holistischen Ansatz eine Leerstelle der anderen hier besprochenen Arbeiten und nimmt neuere Bestrebungen nach einer dekolonialen Wende bezüglich der Betrachtung des Digitalen und seiner Arbeitsverhältnisse vorweg (Casilli, 2017). Zugleich betont er die oft vernachlässigte Materialität des Digitalen. Dabei ist vor allem die Darlegung der Zusammenhänge vermeintlich differenter Phänomene eine Stärke des Buches, auch wenn dabei tiefer gehende Analysen von Arbeit im Digitalen zurück bleiben. Allerdings wirkt sich der Spagat zwischen antagonistischen Theoriesträngen auf den Inhalt des Buches aus. Denn wo die Struktur des kybernetischen Kapitalismus als umfassend und unausweichlich beschrieben wird, erscheinen Widerstände marginal und unwahrscheinlich, so wie sich auch das Jahr 2011 im Rückblick als weniger revolutionär erwies als zunächst angenommen. Und ebenso ist unklar, wie sich das Cyber-Proletariat als „Klasse auf dem Papier“ angesichts solcher Strukturen zu einer tatsächlichen sozialen Realität entwickelt.

\section{Fazit}

Wenig überraschend lässt sich abschließend konstatieren, dass die Schlagrichtung der jeweiligen Perspektive auf den Plattformkapitalismus zentral von der analytischen sowie der theoretischen Position abhängt. Von der Warte der Plattformen und vor allem der Nutzerinnen und Nutzer aus betrachtet erscheinen, wie bei Sundararajan, plattformkapitalistische Phänomene als nachhaltiges zukunftsweisendes Modell, das nicht-ökonomische Aspekte ins Zentrum des Wirtschaftens rückt. Aus Sicht der Arbeitenden stehen die zunehmende Kommodifizierung und der Abbau etablierter Regulationsdispositive im Vordergrund. Nicht unmöglich erscheint, dass beide Sichtweisen begründet sind, doch daraus würde eine sehr ungleiche Erfahrung mit Plattformen folgen, abhängig davon, ob die Beteiligten über oder unter der App verortet sind, ob ihre Arbeit also von der App gesteuert wird oder ob sie selber als Angestellte die Arbeitsprozesse und deren digitale Infrastrukturen organisieren. Und allgemein stehen angesichts 
dieser Art der Arbeitsorganisation die etablierten Formen der Arbeitsbeziehungen auf gesondertem Prüfstand. Denn insofern Outsourcing zur Restrukturierung der Arbeitsbeziehungen sowie der Unterminierung kollektiven Interessenhandelns führt, ist anzunehmen, dass dies für das durch Plattformen ermöglichte Mikro-Outsourcing, bei der noch die kleinste Arbeit ausgelagert werden kann, in besonderem Maße gilt.

Die weithin vertretene These eines anstehenden quasi-revolutionären gesellschaftlichen Wandels infolge der neuen Plattformen ist meist ein Garant für eine hohe Aufmerksamkeit und ist demnach gewiss auch impact-strategisch zu verstehen. Allerdings harrt sie bisher ihrer empirischen Fundierung. Bei näherem Hinsehen stellen sich viele der in diesen Büchern behandelten Phänomene als weit weniger ausgeprägt dar, als der mediale Diskurs vermuten lässt. Nichtsdestotrotz sind Plattformen keine Chimäre, doch bisher sind empirisch fundierte Erkenntnissen über Ausmaß, Ausprägung und Prozesse plattformbasierter Arbeit begrenzt. Während die Existenz von Plattformen vor allem in US-amerikanischen Metropolen in Form von Taxis, Einkaufshilfen, FahrradkurierInnen u.a. augenscheinlich ist, spiegelt sich dergleichen nicht in den verfügbaren offiziellen Statistiken wider. Die vorhandenen Datengrundlagen stoßen mitunter an ihre Grenze, so dass kosten- und zeitaufwendige Erhebungen unausweichlich sind. Und solche sind bisher gering an der Zahl.

Gemein ist den hier diskutierten Büchern, dass sie bezüglich der konkreten Plattformarbeit auf eigene Erhebungen verzichten und auch nur begrenzt auf empirische Erkenntnisse anderer eingehen. Alle vier versuchen sich daran, einen allgemeinen Überblick zu geben, der eher einer , armchair sociology ' gleich kommt. Dies ist durchaus hilfreich, doch ist damit zugleich auch auf die Notwendigkeit fundierter empirischer Arbeiten verwiesen. Eine Übertragung der Annahmen, die für den US-amerikanischen Raum gelten mögen, aber nicht die institutionellen Spezifika des deutschen Kontextes berücksichtigt, erzeugt leicht ein unpassendes Zerrbild. Doch angesichts der medialen wie seit einiger Zeit auch wissenschaftlichen Aufmerksamkeit, die das Phänomen erhält, finden sich fast täglich weitere Veröffentlichungen zur Thematik, deren Anzahl in der nächsten Zeit voraussichtlich noch zunehmen wird. Die Zeichen für ein elaborierteres und fundierteres Verständnis dieser Art der Arbeit und Arbeitsbeziehungen stehen also gut.

\section{Literatur}

Belk, R. (2010). Sharing. Journal of Consumer Research, 36 (5), 715-734. https://doi.org/10.1086/612649

Belk, R. (2014). You are what you can access: Sharing and collaborative consumption online. Journal of Business Research, 67 (8), 1595-1600. doi: 10.1016/j.jbusres.2013.10.001

Benkler, Y. (2004). „Sharing Nicely“: On shareable goods and the emergence of sharing as a modality of economic Production. Yale Law Journal, 114, 273-358. doi: 10.2307/4135731

Bourdieu, P. (1998). Praktische Vernunft. Zur Theorie des Handelns. Frankfurt/Main: Suhrkamp.

Bourdieu, P. (1999). Die Regeln der Kunst. Genese und Struktur des literarischen Feldes. Frankfurt/Main: Suhrkamp.

Casilli, A. A. (2017). Digital Labor Studies Go Global: Toward a Digital Decolonial Turn. International Journal of Communication, 11, 3934-3954.

Duda, J. (2016). Beyond Luxury Cooperativism. In T. Scholz \& N. Schneider (Hrsg.), Ours to Hack and to Own. The Rise of Platform Cooperaticism, a new Vision for the Future of Work and a Fairer Internet (S. 182-186). New York: OR Books. 
Dudenhöffer, K. \& Meyen, M (2012). Digitale Spaltung im Zeitalter der Sättigung. Eine Sekundäranalyse der ACTA 2008 zum Zusammenhang vonInternetnutzung und sozialer Ungleichheit. Publzistik, 57, 7-26. doi: 10.1007/s11616-011-0136-3

Dyer-Whiteford, N. (1999). Cyber-Marx Cycles and Circuits of Struggle in High Technology Capitalism. Urbana: University of Illinois Press.

Fuchs, C. (2014). Digital Labour and Karl Marx. New York: Routledge.

Gillespie, T. (2010). The Politics of 'Platforms'. New Media \& Society, 12 (3), 347-364. doi: $10.1177 / 1461444809342738$

Goodwin, T. (3. März 2015). "The Battle Is For The Customer Interface”. TechCrunch. Abgerufen am 30.01.2018 von

https://techcrunch.com/2015/03/03/in-the-age-of-disintermediation-the-battle-is-all-for-thecustomer-interface/

Harvey, D. (2010). The Enigma of Capital and the Crisis of Capitalism. London: Profile.

Heiland, H. (2018, i.E.). Algorithmus = Logik + Kontrolle. Algorithmisches Management und die Kontrolle der einfachen Arbeit. In D. Houben \& B. Prietl (Hrsg.), Datengesellschaft. Einsichten in die Datafizierung des Sozialen. Bielefeld: Transcript.

Hochschild, A. R. (1983). The Managed Heart: Commercialization of Human Feeling. Berkeley: University of California Press.

Lessig, L. (2009). Remix: Making Art and commerce thrive in the Hybrid Economy. New York: Penguin.

Lobo, S. (3. September 2014). Die Mensch-Maschine: Auf dem Weg in die Dumpinghölle. Abgerufen am 30.01.2018 von http://www.spiegel.de/netzwelt/netzpolitik/sascha-lobo-sharing-economy-wie-bei-uber-istplattform-kapitalismus-a-989584.html.

Luxemburg, R. (1899 [1982]). Sozialreform oder Revolution? In Gesammelte Werke, Bd. 1, Berlin: Dietz.

Penz, O. \& Sauer, B. (2016). Affektives Kapital. Die Ökonomisierung der Gefühle im Arbeitsleben. Frankfurt/Main: Campus.

Sassen, S. (2016). Making Apps for Low-Wage Workers and Their Neighborhoods. In T. Scholz \& N. Schneider (Hrsg.), Ours to Hack and to Own. The Rise of Platform Cooperaticism, a new Vision for the Future of Work and a Fairer Internet (S. 149-153). New York: OR Books.

Schor, J. B. (2017). Does the Sharing Economy Increase Inequality Within the Eighty Percent? Findings from a Qualitative Study of Platform Providers. Cambridge Journal of Regions, 10 (2), 263279. doi: 10.1093/cjres/rsw047

Schor, J. B., Fitzmaurice, C., Carfagna, L. B., Attwood-Charles, W. \& Poteat, E. D. (2016). Paradoxes of Openness and Distinction in the Sharing Economy. Poetics, 54, 66-81. doi: 10.1016/j.poetic.2015.11.001

Sevignani, S. (2017). Facetten der Debatte über das digitale Arbeiten. Herausforderungen für eine kritische Theorie des informationellen Kapitalismus. PROKLA, 47 (1), 43-62.

Srnicek, N. \& Williams, A. (14. Mai 2013). "ACCELERATE MANIFESTO for an Accelerationist Politics". Critical Legal Thinking. Abgerufen am 30.01.2018 von http://criticallegalthinking.com/2013/05/14/accelerate-manifesto-for-an-accelerationist-politics/

Srnicek, N. \& Williams, A. (2015). Inventing the Future: Postcapitalism and a World Without Work. London: Verso.

Staab, P. (2017). The Consumption Dilemma of Digital Capitalism. Transfer, 23 (2), 1-14. doi: $10.1177 / 1024258917702830$

Stanford, J. (2017). The Resurgence of Gig Work: Historical and Theoretical Perspectives. Economic and Labour Relations Review, 28 (3), 382-401. doi: 10.1177/1035304617724303 
Sundararajan, A. (2014). Trusting the "Sharing Economy" to Regulate Itself, New York Times, 3 März 2014, Abgerufen am 30.01.2018 von http://economix.blogs.nytimes.com/2014/03/03/trusting-the-sharing-economy-to-regulate-itself

Wohlsen, M. (12. Mai 2013). Google Pours Millions Into New Tech Gold Rush: Housecleaning. Abgerufen am 30.01.2018 von http://www.wired.com/2013/12/google-homejoy-funding/ 


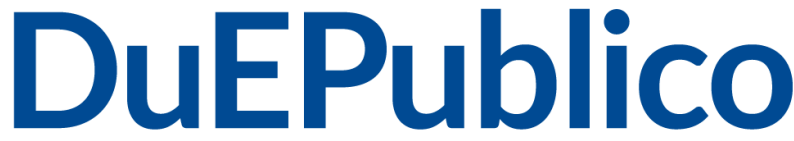

Duisburg-Essen Publications online offen im Denken

$\mathbf{U b} \mid \begin{aligned} & \text { universitäts } \\ & \text { bibliothek }\end{aligned}$

Dieser Text wird über DuEPublico, dem Dokumenten- und Publikationsserver der Universität Duisburg-Essen, zur Verfügung gestellt. Die hier veröffentlichte Version der EPublikation kann von einer eventuell ebenfalls veröffentlichten Verlagsversion abweichen.

DOI: $\quad 10.3224 /$ indbez.v25i1.06

URN: urn:nbn:de:hbz:464-20200702-154318-8

Dieses Werk kann unter einer Creative Commons Namensnennung - Weitergabe unter gleichen Bedingungen 4.0 Lizenz (CC BY-SA 4.0) genutzt werden. 